\title{
A New Retrieval Algorithm Based on Pulse Coupled Neural Network for Biomedical Images
}

\author{
G. Kalaiarasi, K. K. Thyagharajan
}

\begin{abstract}
The rapid expansion and improvement in medical science and technology lead to the generation of more image data in its regular activity such as computed tomography (CT), $X$-ray, magnetic resonance imaging (MRI) etc. To manage the medical images properly for clinical decision making, content-based medical image retrieval (CBMIR) system emerged. In this paper, Pulse Coupled Neural Network (PCNN) based feature descriptor is proposed for retrieval of biomedical images. Time series is used as an image feature which contains the entire information of the feature, based on which the similar biomedical images are retrieved in our work. Here, the physician can point out the disorder present in the patient report by retrieving the most similar report from related reference reports. Open Access Series of Imaging Studies (OASIS) magnetic resonance imaging dataset is used for the evaluation of the proposed approach. The experimental result of the proposed system shows that the retrieval efficiency is better than the other existing systems.
\end{abstract}

Keywords: CBMIR, PCNN, biomedical applications, biological model, time series.

\section{INTRODUCTION}

$T_{1}$ here is a necessity in every field to store the previous records for the future reference. In medical field, database plays a major role. The storage and retrieval of medical images in hospitals is becoming a challenging task due to the increase in images daily. In recent years, the retrieval of stored imaging data from the databases for use in clinical applications, education and research becomes the main focus in medical research. Over the past decades, the medical imaging technology such as CT and MRI help doctors to diagnose diseases with the medical images. Because medical images improve the level of diagnostic accuracy significantly, an increasing number of patients are asked to obtain various medical images from radiology departments to highlight the suspected pathology. Those images are compared to help doctors in acquiring knowledge about each and every patient. By using this knowledge doctors will find similar images that come from different patients. These patients tend to have a major possibility of getting the same disease because they have the similar pathological characteristics in their images. So, finding similar images from the image database will significantly help doctors in finding patients who may get the same disease. And it will

Revised Manuscript Received on 14 November, 2019.

* Correspondence Author

G.Kalaiarasi, Sathyabama Institute of Science and Technology, Chennai, India. Email:kalaikannan.1@gmail.com

K.K.Thyagharajan, R.M.D. Engineering College, Anna University, Chennai, India. Email: kkthyagharajan@yahoo.com also assist doctors to make diagnoses by acquiring information from the previous diagnoses and results. Dermatologists diagnose the skin disease based on the visual assessment of the skin. This shows that correct diagnosis is highly dependent on the physician's experience and on his or her visual perception.

The performance of any image retrieval system is determined by the feature extraction process. The extraction of features is an essential process in image retrieval system where efficiency depends on the method adopted for extracting. The feature vectors are then computed to facilitate the image retrieval process easy. The performance and efficiency of any CBIR system are heavily dependent upon the feature vectors. In this paper, $\mathrm{PCNN}$ is used for feature extraction to improve the performance and is a diagnostics aid to be used by clinicians. The advancement of teleradiology leads to an increasing need for doctors to transmit images to healthcare professionals all over the globe to seek high-quality diagnosis or second opinions. In such situations, our work will be useful for the doctors to provide the second opinion in an easy way. There are many challenges faced in the biomedical image retrieval algorithm. The retrieval accuracy, time complexity, computational cost and lack of efficiency in the existing methods motivated to propose a new methodology for retrieval of biomedical images. Our proposed work is attempted to meet all these challenges.

The remaining section of this paper is organised as follows: the works considering retrieval of medical images in the literature are discussed in section 2, the proposed system is discussed in section 3 , section 4 has the discussion of results and finally, conclusion is presented in section 5 .

\section{RELATED WORKS}

In this section, some of the works related to that of the proposed work including Pulse-Coupled Neural Network and CBIR in Medical Images is discussed.

\section{A. Pulse Coupled Neural Network}

PCNN is good at digital image processing. The related models and its applications are discussed in [1]. Wang et al. presented the corresponding review of research studies [2]. They discussed the current status of the PCNN, different models including intersecting cortical model (ICM), unit-linking PCNN model, multi-channel PCNN model and their applications in the image processing field. Salient region detection is applied in many processes such as object detection, image compression, image registration, etc. 
PCNN is well-known for the way of implementation, simplicity, accuracy in recognition and anti-noise disturbance. Also, PCNN is used for texture segmentation, fabric defect analysis and surveillance techniques. It is a good pre-processor and can be applied to remove the noise present in images [3]. Dual Channel PCNN algorithm has been developed for the fusion of multimodality brain images [4]. Optimised PCNN in along with K-Nearest Neighbor (K-NN) is used for classification and retrieval of images by extracting visual features known as image signature [5]. Quantum based Particle Swarm Optimization along with adaptive PCNN fuses multimodal medical images [6]. Genetic algorithm is used in image fusion for optimal parameter estimation [7]. Generally, PCNN is used for reducing the number of features.

\section{B. CBIR IN MEDICAL IMAGES / APPLICATIONS}

Some of the works related to retrieval of images is discussed here. Marko et al. presented center-symmetric LBP (CS-LBP) in [8]. CS-LBP is the combination of scale invariant feature transform (SIFT) and LBP. An uncertain location graph (ULG) is presented for the modelling and similarity retrieval of brain CT images in [9]. This method is best suited as it resulted in higher accuracy and efficiency. But the ULG-matching time is more. Murala et al. compared biomedical image retrieval algorithms that used local mesh pattern (LMeP) with other existing algorithms such as local binary pattern (LBP) and other spatial - transform domain methods in [10]. The similarity and difference between the referenced pixel and the surrounding neighbouring pixels is encoded by LBP. But LMeP encodes the similarity and difference between the surrounding neighbours of a particular pixel and that particular pixel of an image. The execution time of LMeP is more and the feature vector length is also more even though the results outperform other existing methods. CBIR system diagnosing the skin lesion images is presented in [11]. The similar images will help the dermatologists to take clinical decision. But there are more false positives and false negatives. The local bit-plane decoded pattern (LBDP) is used for indexing and retrieval of biomedical images in [12]. The local mesh ternary pattern (LMeTerP) and directional local ternary quantized extrema pattern (DLTerQEP) is proposed by Gagan et al. [13]. To improve the retrieval accuracy in the biomedical MRI images, our proposed system uses PCNN to extract the features.

\section{PROPOSED SYSTEM}

In this proposed work, a novel methodology is proposed for retrieval of biomedical images. First features are extracted from the images stored in the dataset. Then similarity among the images present in the database is computed. Based on the similarity value, decisions are made for any medical diagnosis.

\section{A. OBJECTIVE}

The primary objective of this work is to retrieve similar images for a particular query image. This helps doctor/physician to point out the disorder present in the patient report by retrieving the most similar report from related reference reports.

\section{B. MATERIALS AND METHODS}

Pulse Coupled Neural Network was proposed by modeling a cat's visual cortex. The functioning of PCNN is inspired by biological neuron model. The number of neurons present in the network depends on the number of pixels in the image. It is a single layered and two-dimensional neural network. The neuron is compose of a feeding field, a linking modulation and a pulse generator.

The input signals from neighbouring neurons and from external sources are sent to feeding field. Linking field receives input from the neighbouring neurons. The Modulation part is obtained by multiplying feeding input with biased and linking inputs to form total internal activity U. A step function generator and a threshold signal generator are present in pulse generator part. The output $\mathrm{Y}$ is set to 1 when the internal activity of the neuron exceeds a certain threshold and becomes zero when the threshold is larger than the internal activity $U$. The pulse outputs are produced by PCNN for $\mathrm{n}$ number of iterations. This output has certain information of the input image. The weight $\mathrm{M}$ and $\mathrm{W}$ are used to communicate between the surrounding neurons. The block diagram of PCNN is given in figure 1.

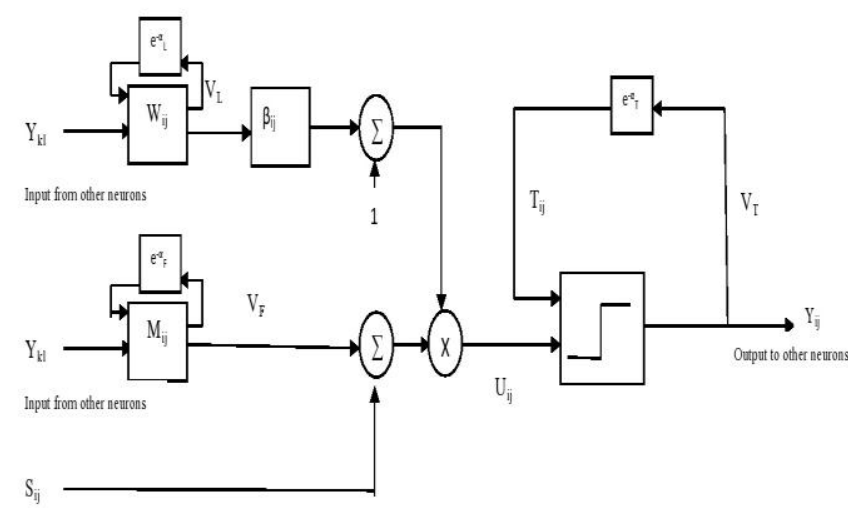

Fig. 1.Block Diagram of PCNN

PCNN can be implemented using following equations:

$$
\begin{aligned}
& F_{i j}[n]=S_{i j}+F_{i j}[n-1] e^{-\alpha_{F}}+V_{F} \sum_{k l} M_{i j k l} Y_{k l}[n-1] \\
& L_{i j}[n]=L_{i j}[n-1] e^{-\alpha_{L}}+V_{L} \sum_{k l} W_{i j k l} Y_{k l}[n-1] \\
& U_{i j}[n]=F_{i j}[n]\left(1+\beta L_{i j}[n]\right) \\
& T_{i j}[n]=T_{i j}[n-1] e^{-\alpha_{T}}+V_{T} Y_{i j}[n-1] \\
& Y_{i j}[n]=\left\{\begin{array}{cc}
1, & \text { if } U_{i j}[n]>T_{i j}[n] \\
0, & \text { else }
\end{array}\right.
\end{aligned}
$$

In these equations, $(i, j)$ is the position of a neuron in the network and a pixel in the image. $(k, 1)$ refers the positions of the surrounding neurons; $n$ is the iteration number, and $S_{i j}$ is the 
gray level of the input image. The $\mathrm{F}_{\mathrm{ij}}[\mathrm{n}], \mathrm{L}_{\mathrm{ij}}[\mathrm{n}], \mathrm{U}_{\mathrm{ij}}[\mathrm{n}]$ and $\mathrm{T}_{\mathrm{ij}}[\mathrm{n}]$ refers to the feeding input, linking input, internal activity and dynamic threshold respectively. The $\mathrm{Y}_{\mathrm{ij}}[\mathrm{n}]$ is the binary value that refers the output of the neuron. $\mathrm{M}$ and $\mathrm{W}$ are the constant weight matrices. The linking coefficient is $\beta$, the attenuation time constants are $\alpha_{\mathrm{F}}, \alpha_{\mathrm{L}}, \alpha_{\mathrm{T}}$ and $\mathrm{V}_{\mathrm{F}}, \mathrm{V}_{\mathrm{L}}, \mathrm{V}_{\mathrm{T}}$ are the voltage potential of the feeding signal, linking signal and dynamic threshold respectively.

\section{FEATURE EXTRACTION AND SIMILARITY COMPUTATION}

A series of binary images are computed from the input image by PCNN. These binary images contain lots of information that is features of the original image. But these features can't be used directly as it is too big. Some kind of transformations can be carried out on these sequence images to extract a small amount of data as an original image feature. Johnson [1] first proposed the time series $G(n)=$ $\sum_{n=1}^{N} Y(n)$, as image feature where $\mathrm{n}$ is the iteration $(n=1, \ldots N)$. The length of the feature vector is based on the total number of the PCNN iterative steps. After image feature extraction, Correlation Coefficient is used to compute the similarity between two images. Suppose feature vectors of two images are $\mathrm{x}_{\mathrm{i}}=\left[\mathrm{x}_{\mathrm{i} 1}, \mathrm{x}_{\mathrm{i} 2}, \ldots \mathrm{x}_{\mathrm{iN}}\right]$ and $\mathrm{x}_{\mathrm{j}}=\left[\mathrm{x}_{\mathrm{j} 1}, \mathrm{x}_{\mathrm{j} 2}, \ldots \mathrm{x}_{\mathrm{jN}}\right]$ respectively, $\mathrm{CC}$ is defined as

$C C=\frac{\sum_{a=1}^{N}\left(x_{i a}-\mu_{i}\right)\left(x_{j a}-\mu_{j}\right)}{\sqrt{\sum_{a=1}^{N}\left(x_{i a}-\mu_{i}\right)^{2} X \sum_{a=1}^{N}\left(x_{j a}-\mu_{j}\right)^{2}}}$

where $N$ is the dimension of feature vector, $\mu_{i}$ and $\mu_{j}$ separately indicate the mean of vector $x_{i}$ and $x_{j}$ as shown below -

$\mu_{i}=\frac{1}{N} \sum_{a=1}^{N} x_{i a}, \mu_{j}=\frac{1}{N} \sum_{a=1}^{N} x_{j a}$

Generally, if the value of CC is closer to 1 , then there is a greater linear correlation of the two feature vectors, that is, higher similarity between two images; if the $\mathrm{CC}$ is closer to 0 , the smaller correlation among the two vectors, that is, lower similarity among the images.

\section{PCNN - MIR ALGORITHM}

Input: Images $\mathrm{I}_{\mathrm{i}}$, where $\mathrm{i} \epsilon 1,2 \ldots, \mathrm{n}$

Output: Similarity value between the images

1: Read the image

2: Store rowsize, colsize

3: Assign the pixel value to $S(i, j)$

4: Initialize PCNN parameters: $\mathrm{Y}, \mathrm{G}, \mathrm{T} \leftarrow$ zeros(rowsize, colsize)

5: Set the PCNN parameters M, W, aF, aL, aT, $\mathrm{V}_{\mathrm{F}}, \mathrm{V}_{\mathrm{L}}, \mathrm{V}_{\mathrm{T}}, \mathrm{b}$

6: Set number of iterationsnum

7: for $n \in\{1$, num $\}$ do

8: Compute sum $1 \leftarrow$ imfilter $(\mathrm{Y}, \mathrm{M})$

9:

sum $2 \leftarrow$ imfilter(Y, W)
10: for $\mathrm{i} \in\{1$, rowsize $\}$ do

11: for $\mathrm{j} \epsilon\{1$, colsize $\}$ do

12: $\quad \mathrm{F}(\mathrm{i}, \mathrm{j}) \leftarrow \mathrm{aF}^{*} \mathrm{~F}(\mathrm{i}, \mathrm{j})+\mathrm{S}(\mathrm{i}, \mathrm{j})+\mathrm{V}_{\mathrm{F}}{ }^{*} \operatorname{sum} 1(\mathrm{i}, \mathrm{j})$;

13: $\quad \mathrm{L}(\mathrm{i}, \mathrm{j}) \leftarrow \mathrm{aL} * \mathrm{~L}(\mathrm{i}, \mathrm{j})+\mathrm{V}_{\mathrm{L}} * \operatorname{sum} 2(\mathrm{i}, \mathrm{j})$;

14: $\quad \mathrm{U}(\mathrm{i}, \mathrm{j}) \leftarrow \mathrm{F}(\mathrm{i}, \mathrm{j}) *\left(1+\mathrm{b}^{*} \mathrm{~L}(\mathrm{i}, \mathrm{j})\right) ;$

15: $\quad \mathrm{T}(\mathrm{i}, \mathrm{j}) \leftarrow \mathrm{aT}^{*} \mathrm{~T}(\mathrm{i}, \mathrm{j})+\mathrm{V}_{\mathrm{T}} * \mathrm{Y}(\mathrm{i}, \mathrm{j}) ;$

16: $\quad$ if $U(i, j)-E(i, j)>0$

17: then

18: $\quad \mathrm{Y}(\mathrm{i}, \mathrm{j}) \leftarrow 1$;

19: $\quad$ else

20: $\quad \mathrm{Y}(\mathrm{i}, \mathrm{j}) \leftarrow 0$;

21: for $n \in\{1$, num $\}$ do

22: for $i \epsilon\{1$, rowsize $\}$ do

23: for $\mathrm{j} \in\{1$, colsize $\}$ do

24: $\quad \mathrm{G}(\mathrm{n}) \leftarrow \mathrm{G}(\mathrm{n})+\mathrm{Y}(\mathrm{i}, \mathrm{j})$

25: Repeat the same steps for all the images

26: Compute Similarity using Correlation Coefficient

27: $\mathrm{r} \leftarrow \operatorname{Corr}(\mathrm{A}, \mathrm{B})$ where $\mathrm{A}, \mathrm{B} \in \mathrm{G}$ value of images

\section{RESULTS AND DISCUSSION}

\section{A. PARAMETER SETTING}

Parameter setting is an essential step as it will affect the output. It varies based on the application. But for a specific application all the images must use same parameters for PCNN. Based on trial and error method, the parameters are set in advance as shown in the Table I. L, U and Y matrices are initially set to zero and Connection Matrix $\mathrm{W}$ and $\mathrm{M}$ is given by $[0.5,1,0.5 ; 1,0,1 ; 0.5,1,0.5]$.

TABLE I. PARAMETERS OF PCNN

\begin{tabular}{|c|c|c|c|c|c|c|c|}
\hline Parameters & $\boldsymbol{\alpha}_{\mathbf{F}}$ & $\boldsymbol{\alpha}_{\mathbf{L}}$ & $\boldsymbol{\alpha}_{\mathbf{T}}$ & $\mathbf{V}_{\mathbf{F}}$ & $\mathbf{V}_{\mathbf{L}}$ & $\mathbf{V}_{\mathbf{T}}$ & $\mathbf{b}$ \\
\hline Values & 0.1 & 1.0 & 1.0 & 0.5 & 0.2 & 20 & 0.1 \\
\hline
\end{tabular}

These values are used for processing of all the images present in the image set. The values of parameters are sample values. Users can adjust these values according to practical demands. The same can be tried using different parameters also.

\section{B. Dataset}

In this paper, OASIS database is considered for analysis purpose. The OASIS dataset is made public for research and is available online for use in medical research field [14] [15]. A cross-sectional collection of 421 patients between 18 and 96 years of age is used for the analysis of the proposed work. The resolution of those images is 176 X 208. Further, based on the ventricular shape inside the images, the categories formed from 421 images are C1 (124 images), C2 (102 images), C3 (89 images) and C4 (106 images). Fig. 2 depicts the sample images for each category. 


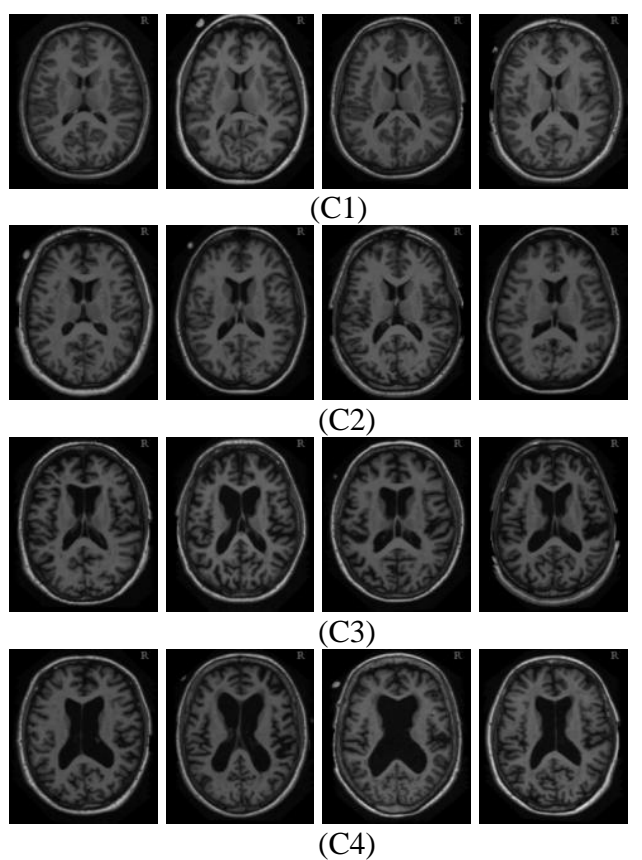

Fig. 2.OASIS-MRI sample images - four images from each category $\mathrm{C} 1, \mathrm{C} 2$, $\mathrm{C} 3$, and $\mathrm{C} 4$

First, features are extracted using PCNN. Next, the similarity is computed using correlation coefficient. Here, all the images in the database are considered as the query image. For each query, the system collects images with more correlation. If these images are from the same category of the query image, then the system has correctly matched the desired. For the query image, the precision $(\mathrm{P})$ and recall $(\mathrm{R})$ are calculated using the following:

$$
\begin{aligned}
& \mathrm{P}=\frac{\text { Number of Relevant images Retrieved }}{\text { Total Number of Images Retrieved }} \\
& \mathrm{R}=\frac{\text { Number of Relevant images Retrieved }}{\text { Total Number of Relevant Images in the database }}
\end{aligned}
$$

Initially, an image from category 1 is given as the query to retrieve the similar images using our algorithm. The proposed PCNN-MIR method computes similarity between the images using correlation coefficient and retrieves the relevant images from the dataset. Then, a query image is taken from category 2, 3 and 4. Based on the images retrieved, the corresponding values are computed and shown in Table II \& Table III.

TABLE II. CATEGORY-WISE PRECISION VALUE

\begin{tabular}{|c|c|c|c|}
\hline Category & $\begin{array}{c}\text { No. of Relevant } \\
\text { Images Retrieved }\end{array}$ & $\begin{array}{c}\text { Total No. of Images } \\
\text { Retrieved }\end{array}$ & Precision \\
\hline C1 & 70 & 139 & 50.36 \\
\hline C2 & 76 & 135 & 56.29 \\
\hline C3 & 75 & 105 & 71.43 \\
\hline C4 & 97 & 167 & 58.08 \\
\hline \multicolumn{3}{|c|}{ Average Precision } \\
\hline
\end{tabular}

TABLE III. CATEGORY-WISE RECALL VALUE

\begin{tabular}{|c|c|c|c|}
\hline Category & $\begin{array}{c}\text { No. of Relevant } \\
\text { Images Retrieved }\end{array}$ & $\begin{array}{c}\text { Total No. of } \\
\text { Relevant Images in } \\
\text { DataBase }\end{array}$ & Recall \\
\hline C1 & 70 & 124 & 56.45 \\
\hline C2 & 76 & 102 & 74.51 \\
\hline C3 & 75 & 89 & 84.26 \\
\hline C4 & 97 & 106 & 91.51 \\
\hline \multicolumn{3}{|c}{ Average Recall } \\
\hline
\end{tabular}

TABLE IV. COMPARISON OF VARIOUS TECHNIQUES ON OASIS-MRI DATABASE.

\begin{tabular}{|c|c|}
\hline Method & Precision (\%) \\
\hline LBPu2_8_1 & 42.63 \\
\hline LBPu2_16_2 & 44.37 \\
\hline LmeTerP & 44.47 \\
\hline CS_LBP & 41.06 \\
\hline BLK_LBP & 43.63 \\
\hline DBWP & 47.05 \\
\hline LTP & 45.17 \\
\hline DLEP & 48 \\
\hline LDP & 41.8 \\
\hline LmeP & 44.96 \\
\hline LQEP & 40.51 \\
\hline DLTerQEP & 48.19 \\
\hline PCNN & 59.04 \\
\hline
\end{tabular}

Given below are the abbreviations used in the analysis of result:

LBP_P_Ru2: Local binary pattern features collected from uniform two pattern size $(\mathrm{P}, \mathrm{R})$

LMeTerP: Local Mesh Ternary Pattern

CSLBP: Center-symmetric LBP

BLK_LBP: Block based LBP

DBWP: Directional binary wavelet patterns

LTP: Local Ternary Pattern

DLEP: Directional Local Extrema Pattern

LDP: Local Derivative Pattern

LMeP: Local mesh patterns

\section{LQeP: Local Quantized Extrema Pattern}

DLTerQEP: Directional Local Ternary Quantized Extrema Pattern

Table IV summarises the retrieval results of PCNN and other existing methods [13] on OASIS-MRI image database in terms of Precision for biomedical image retrieval. The recall value is $76.68 \%$.

\section{CONCLUSION}

This paper mainly deals with CBIR in a biomedical application using PCNN. In this paper, PCNN based feature descriptor is proposed for retrieval of biomedical images. The results prove that our work outperforms the existing biomedical image retrieval approaches. Hence, the physician can point out the disorder present in the patient report retrieving the most similar report from related reference reports. This is used in many other biomedical applications for diagnosis, education, and research.

\section{REFERENCES}

Johnson, J.L., Padgett, M.L., 1999, May. PCNN models and applications IEEE transactions on neural networks. 10(3), pp.480-98.

Wang, Z., Ma, Y., Cheng, F., Yang, L., 2010, Jan. Review of pulse-coupled neural networks. Image and Vision Computing. 28(1),pp.5-13.

Subashini, M.M., Sahoo, S.K., 2014, June. Pulse coupled neural network and its applications. Expert Systems with Applications. 41(8),pp.3965-74 
4. Kavitha, S., Thyagharajan, K.K., 2014, July. Dual channel Pulse Coupled Neural Network algorithm for fusion of multimodality brain Images with quality analysis. Applied Medical Informatics. 35(3),pp.31.

5. Mohammed, M.M., Badr, A., Abdelhalim, M.B., 2015, July. Image classification and retrieval using optimized pulse-coupled neural network. Expert systems with applications. 42(11),pp.4927-36.

6. Xu, X., Shan, D., Wang, G., Jiang, X., 2016. Multimodal medical image fusion using PCNN optimized by the QPSO algorithm. Applied Soft Computing. 46,pp.588-95.

7. Kavitha, S., Thyagharajan, K.K., 2016. Efficient DWT-based fusion techniques using genetic algorithm for optimal parameter estimation. Soft Computing. 1-0.

8. Heikkila, M., Pietikainen, M., Schmid, C., 2009, Mar. Description of interest regions with local binary patterns. Pattern recognition. 42(3),pp.425-36

9. Pan, H., Li, P., Li, Q., Han, Q., Feng, X., Gao, L., 2014, Mar. Brain CT image similarity retrieval method based on uncertain location graph. IEEE journal of biomedical and health informatics. 18(2),pp.574-84.

10. Murala, S., Wu, Q.J., 2014, May. Local mesh patterns versus local binary patterns: biomedical image indexing and retrieval. IEEE Journal of Biomedical and Health Informatics. 18(3),pp.929-38.

11. Jiji, G.W., Raj, P.S., 2014, Oct. Content-based image retrieval in dermatology using intelligent technique. IET Image Processing. 9(4), pp.306-17.

12. Dubey, S.R., Singh, S.K., Singh, R.K., 2016, Jul. Local bit-plane decoded pattern: A novel feature descriptor for biomedical image retrieval. IEEE Journal of Biomedical and Health Informatics. 20(4),pp.1139-47.

13. Deep, G., Kaur, L., Gupta, S., 2016, Dec. Biomedical Image Indexing and Retrieval Descriptors: A Comparative Study. Procedia Computer Science. 31(85),pp.954-61.

14. Marcus, D.S., Wang, T.H., Parker, J., Csernansky, J.G., Morris, J.C., Buckner, R.L., 2007, Sep. Open Access Series of Imaging Studies (OASIS): cross-sectional MRI data in young, middle aged, nondemented, and demented older adults. Journal of cognitive neuroscience. 19(9):1498-507.

15. http://www.oasis-brains.org/

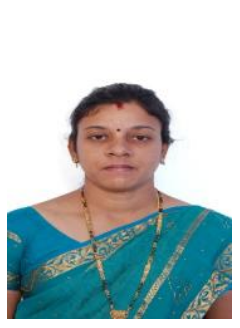

\section{AUTHORS PROFILE}

G.Kalaiarasi has received her B.Tech., degree in Information Technology from Srinivasa Institute of Engineering \& Technology affiliated to Madras University. She has completed M.E., in Computer Science \& Engineering in Dhanalakshmi Srinivasan Engineering College which is affiliated to Anna University. She has completed her Ph.D in Information \& Communication Engineering, Anna University, Chennai. She has published papers in National \& International Journals and Conferences. Her research areas are Computer Vision, Image Processing, Image Retrieval and Data Mining. She has teaching experience of around 8 years and currently, she is working in Sathyabama Institute of Science and Technology, Chennai.

E-mail: kalaikannan.1@gmail.com

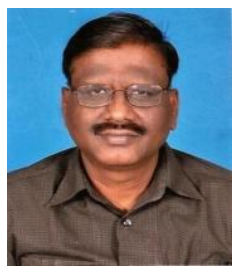

K .K. Thyagharajan B.E,M.E,Ph.D, received his B.Eng. degree in Electrical and Electronics Engineering from PSG College of Technology, Madras University, India and received his M.Eng. degree in Applied Electronics from Coimbatore Institute of Technology, India in 1988. He also possesses a Post Graduate Diploma in Computer Applications from Bharathiar University, India. He obtained his Ph.D. degree in Information and Communication Engineering from College of Engineering Guindy, Anna University, India. He is in teaching profession for around three decades and served at various levels including Principal, Professor and Dean at various Engineering Colleges. He has written 5 books in Computing including "Flash MX 2004" published by McGraw Hill (India), which has been recommended as text and reference book by universities. He is a grant recipient of Tamil Nadu State Council for Science and Technology. He has been invited as chairperson and delivered special lectures in many National and International conferences and workshops. He is reviewer and editorial board member for many International Journals and Conferences. $\mathrm{He}$ is a recognized supervisor for Ph.D candidates and Master students at Anna University. He has published more than 90 papers in National \& International Journals and Conferences. His research interests include Computer Vision, Semantic Web, Image \& Video Processing, Multimedia Streaming and e-learning. He is a life member of ISTE, CSI INDIA and also senior member and invited member in many professional associations. He has been recognized by Marquis Who's Who in the World for his contribution to the technical society and his biography has been published in its 25th Anniversary Edition. Personal. 